\title{
Electron Energy Loss of Terrylene Deposited on Au(111): Vibrational and Electronic Spectroscopy
}

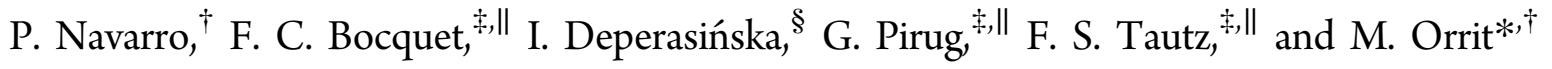 \\ ${ }^{\dagger}$ Huygens-Kamerlingh Onnes Laboratory, University of Leiden, 2300 RA Leiden, Netherlands \\ ${ }^{\ddagger}$ Peter Grünberg Institut (PGI-3), Forschungszentrum Jülich, 52425 Jülich, Germany \\ "Jülich-Aachen Research Alliance (JARA), Fundamentals of Future Information Technology, 52425, Jülich, Germany \\ ${ }^{\S}$ Institute of Physics, Polish Academy of Sciences, 02-668, Warsaw, Poland
}

Supporting Information

\begin{abstract}
We have investigated the vibrational and electronic properties of terrylene by high-resolution electron energy-loss spectroscopy (HREELS), Fourier-transform infrared spectroscopy, and low-temperature single-molecule fluorescence spectroscopy. Terrylene thin films were sublimated in an ultrahigh vacuum on the $\mathrm{Au}(111)$ surface in order to record the HREEL spectra. Polycrystalline $p$ dichlorobenzene was used as a matrix to isolate a single terrylene molecule at $1.5 \mathrm{~K}$ and record its fluorescence spectrum. The infrared spectrum, the vibrational components from the fluorescence spectrum, and density functional theory calculations were used for the assignment and identification of the active modes found in HREELS. Finally, we report a loss signal around $17000 \mathrm{~cm}^{-1}(2.1 \mathrm{eV})$ for the first singlet electronic excited state in agreement with optical spectroscopy. The HREEL spectra show both IR- and Raman-active vibration modes because of specific surface selection rules. Energy-loss spectroscopy could be used as a complementary technique to explore some other degrees of freedom that are not accessible by optical means.

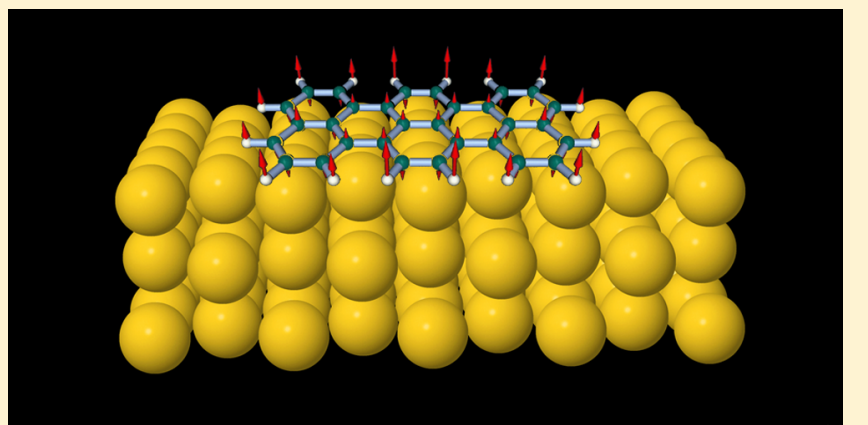

\section{INTRODUCTION}

High-resolution electron energy-loss spectroscopy (HREELS) is a valuable method to characterize organic thin films because it is highly surface sensitive and induces little radiation damage. ${ }^{1}$ From the spectral features in HREELS, it is possible to learn about the physical interactions at the interface between semiconductor materials and metallic surfaces that are crucial in the performance of electronic devices. Information about the orientation of molecules in organic thin films, such as Langmuir-Blodgett films and polymer films, can also be obtained. Because of the penetration depth of the impinging electrons, in general loss signals bring information about molecular degrees of freedom that are excited at the surface or at the interface. Many HREELS studies are focused on the vibrational degrees of freedom of the selected molecules. ${ }^{2}$ However, the electronic properties of organic semiconductor films $^{3,4}$ (molecular exciton band gap) have also been measured with HREELS, using an electron beam with moderately high energy (10 eV or higher) as excitation and looking for energy losses in the range of $1-5 \mathrm{eV}\left(8000-40000 \mathrm{~cm}^{-1}\right)$ where these transitions occur. These observations can be compared with optical spectroscopy in solution. ${ }^{5}$ There have been also some reports of the observation of low-lying triplet states from organic semiconductor films, in particular naphthalene, ${ }^{6}$ and therefore, HREELS seems to be a promising tool to determine the unknown lowest triplet state of terrylene.

Organic semiconductors based on polyaromatic hydrocarbons (PAHs) have been used in the development of novel molecular electronic and photonic devices, such as organic light emitting diodes (OLEDs), photovoltaic solar cells, optical switches, and field-effect transistors. ${ }^{7-9}$ The performance of these devices are determined by the complex combination of electronic and structural properties of the materials. ${ }^{10}$ For example, the electronic band gap of the semiconductor determines the electron and hole mobility ${ }^{11}$ in organic fieldeffect transistors (OFETs). The interfacial and intermolecular interactions are strong driving forces that influence the final structure of the film and therefore play an important role in the properties of the electronic devices like LEDs. ${ }^{12-15}$

The present terrylene ( $\mathrm{Tr}$ ) molecule has a maximum absorption peak (optical spectroscopy) at the center of the visible range, around $570 \pm 10 \mathrm{~nm}$, which corresponds to $2.1 \pm$ $0.04 \mathrm{eV}$. This band gap is larger than the band gap on silicon, 1 $\mathrm{eV}$, which would allow terrylene to be used as a semiconducting medium in molecular electronic devices. Terrylene has a large

Received: August 26, 2014

Revised: December 3, 2014

Published: December 3, 2014 
optical extinction coefficient, $\varepsilon \approx 10^{5} \mathrm{M}^{-1} \mathrm{~cm}^{-1}$, and therefore can be used as an absorbing material in new photovoltaic devices. At the same time, it has a fluorescence quantum yield close to unity, that could be interesting for OLEDs. It is very photostable when embedded in solid matrices ${ }^{16}$ and/or polymers. ${ }^{17}$ The undistorted terrylene molecule belongs to $D_{2 h}$ symmetry, and its planar structure, similar to that of perylene, ${ }^{18-20}$ makes it an interesting candidate for growing ordered films. Thanks to some of these optical, electronic, and structural properties, $\operatorname{Tr}$ seems to be suitable for explore applications as a reddish-pigment for LEDs or as a semiconductor medium for OFETs. ${ }^{11}$

The other very important element in the design of semiconductor devices is the selection of a metallic substrate. Gold has been commonly used as a contact material for source and drain electrodes in OFETs. It is one of the noble metals used as a substrate that presents moderate physisorption interactions with poly aromatic molecules like terrylene. This is an important property to keep the vibrational and the electronic properties of the deposited molecule as unperturbed as possible. ${ }^{4}$ Thus, as the main part of this work we present the characterization of terrylene films deposited under ultrahigh vacuum (UHV) on an $\mathrm{Au}(111)$ surface. We employ surfacesensitive techniques, essentially HREELS, assisted with X-ray photoelectron spectroscopy (XPS).

A further aim of this study was the determination of the energy gap between the ground state and the lowest triplet excited state of terrylene $\left(S_{0} \rightarrow T_{1}\right)$, as has been done for naphthalene using HREELS. ${ }^{6}$ Knowledge of this transition energy would allow us to switch off the continuous fluorescence photon stream from a single terrylene molecule by optical means, by exciting the molecule into this triplet state. ${ }^{21}$ In the case of $\operatorname{Tr}$, the energy for the singlet excited state $\left(S_{1}\right)$ is wellknown. ${ }^{22}$ However, to our knowledge, the energy of the lowest triplet state $\left(T_{1}\right)$ has never been measured. The main difficulty in this optical measurement stems from the weakness of the population yield of $\mathrm{T}_{1}$ from the excited singlet state and from the extremely weak yield of the radiative decay (phosphorescence) from the triplet state to the ground singlet state.

We first report the vibrational spectroscopy of terrylene by HREELS. We use different complementary techniques to analyze and discuss the observations. We used Fourier transform infrared absorption (FTIR) in a $\mathrm{KBr}$ pellet doped with $\operatorname{Tr}$ in a commercial spectrometer. Quantum chemical calculations of terrylene vibrations were performed with the density functional theory (DFT) B3LYP/6-31G(d,p) method. We recorded the fluorescence spectrum from a single terrylene molecule in $p$-dichlorobenzene $(p-\mathrm{DCB})$ at $1.5 \mathrm{~K}^{23}$ The IR spectra showed almost a one-to-one correspondence with the HREEL spectrum. However, some fluorescent active modes could also be observed in the HREEL spectrum. Finally, we studied the electronic properties of terrylene films by HREELS and compared them to optical spectroscopy.

\section{EXPERIMENTAL SECTION}

All HREELS experiments were performed in a home-built UHV chamber at a base pressure of $3.0 \times 10^{-10} \mathrm{mbar}$. A clean $\mathrm{Au}(111)$ surface was produced by repeated $\mathrm{Ar}^{+}$sputtering cycles and subsequent annealing at $650{ }^{\circ} \mathrm{C}$. The cleanliness of the surface was determined by Auger electron spectroscopy (AES, from $80-560 \mathrm{eV}$, data not shown) using a single-pass cylindrical mirror analyzer (CMA) with a coaxial electron gun at $2.7 \mathrm{kV}$ (Varian CMSS-981). The crystallinity of the $\mathrm{Au}(111)$ was characterized by LEED showing the expected $(23 \times \sqrt{ } 3)$ reconstruction as expected for a clean surface. ${ }^{24}$ The incident beam energy used was $E_{0}=45 \mathrm{eV}$ (data not shown). Terrylene powder purchased from Chiron, AS (purity 99\%) was directly inserted in the Knudsen cell that was separately pumped to $3 \times$ $10^{-10}$ mbar. Then, it was treated for $24 \mathrm{~h}$ at $373 \mathrm{~K}$ residual pressure of $3 \times 10^{-8} \mathrm{mbar}$ to be outgassed, dried, and to eliminate possible carbon-containing compounds of lower molecular weight. The optimal sublimation parameters for terrylene in $\mathrm{Au}(111)$ were $469 \pm 4 \mathrm{~K}\left(196 \pm 4{ }^{\circ} \mathrm{C}\right)$ at a residual pressure of $8 \times 10^{-8} \mathrm{mbar}$. Exposure times of $\sim 2 \mathrm{~min}$ led to a controlled deposition rate. After each exposure, X-ray photoelectron spectroscopy (XPS) and HREELS were recorded one after the other. The $K$-alpha radiation from a Mg source $(h \nu=$ $1253.6 \mathrm{eV}$ ) impinges the surface at $-20^{\circ}$, and the electrons are collected by the Leybold-Heraeus spectrometer under $+20^{\circ}$ relative to the surface normal. The HREELS experiments were performed with a home-built spectrometer in specular geometry, $\theta_{\text {in }}=\theta_{\operatorname{det}}=70^{\circ}$. The incident beam energy was set at $E_{0}=5.0 \mathrm{eV}$ for vibrational spectroscopy and $12 \mathrm{eV}$ for electronic excitation. The resolution of the spectrometer determined by measuring the fwhm of the peak at zero-loss energy was 1.2 and $5 \mathrm{meV}\left(10\right.$ and $40 \mathrm{~cm}^{-1}$ ) for the direct and the elastically scattered beam, respectively.

The complete fluorescence spectrum from a single terrylene has been measured using a poly crystal of $p-\mathrm{DCB}$ as a hostmatrix at $1.5 \mathrm{~K}^{23}$ The Fourier-transform infrared spectrum was measured in a commercial FTIR-spectrometer. The sample was a very fine powder of $\mathrm{Tr}$ mixed with $\mathrm{KBr}$ as excipient and then was pressed to form a thin pellet. All calculations in this work were done with the Gaussian-09 package. ${ }^{25}$ Optimization of the molecular geometry in the electronic ground and lowest excited states were done with the DFT and TDDFT B3LYP/6$31 \mathrm{G}(\mathrm{d}, \mathrm{p})$ methods. Vibrational structure of the fluorescence spectrum was calculated with a procedure included into Gaussian-09. ${ }^{26}$

\section{RESULTS AND DISCUSSION}

3.1. Vibrational Spectroscopy of Terrylene. When an incident electron beam impacts the surface, the molecule has a probability to be excited to one of the vibrational levels of the ground state. As a result, the scattered electron transfers to the molecule some of its initial energy giving rise to the recorded energy-loss signal in the spectrum.

As a result of the excitation, an oscillating motion of the atoms of the deposited molecule induces a polarization of the gold electrons, creating a surface dipole. For molecular vibrations with atomic displacements perpendicular to the surface plane (out-of-plane modes), the electrostatic images enhance the dipole moment and the transition strength (left side of Chart 1). For molecular vibrations with atomic displacements parallel to the surface (in-plane modes), the electrostatic images suppress the dipole moment (right side of Chart 1). As a result, the intensities of the out-of-plane vibrations are enhanced by the induced surface dipole, while those of the in-plane vibrations are strongly suppressed as will be discussed below.

Figure 1 shows the HREEL (Figure 1a), the measured (Figure 1b) and the calculated (Figure 1c) infrared absorption, and the fluorescence (Figure 1d) spectra of Tr measured in the vibrational region from 0 to $3500 \mathrm{~cm}^{-1}$. In the following, we discuss each of the modes appearing in the HREEL spectrum of a thin film of terrylene (Figure 1a) with increasing frequency 
Chart 1. Effect of Electrostatic Image Charges in the Metallic Surface on the Dipole Moments of Molecular Vibrations ${ }^{a}$

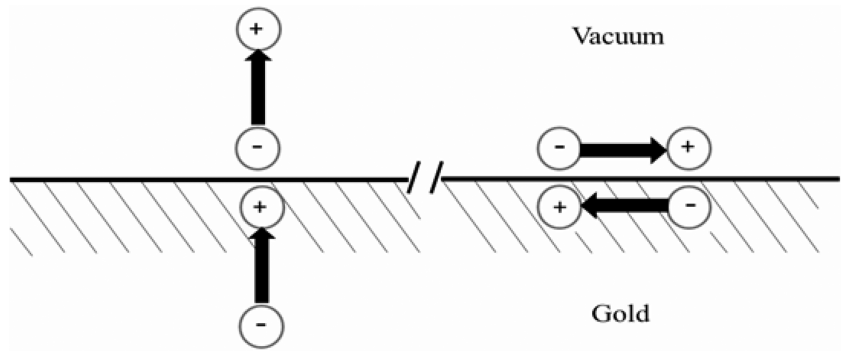

${ }^{a}$ Left: out-of-plane dipole moments are strengthened by the surface image dipole, enhancing the Coulomb interactions with impinging electrons. Right: for in-plane dipole moments, the image charges shields the field on impinging electrons and the Coulomb interaction with the mode is strongly suppressed.

and compare them to the other spectra. The HREEL spectra were always measured together with XPS after each exposure.

The first loss signal on the wing of the elastic peak appears at $185 \mathrm{~cm}^{-1}$. Although this region was not accessible to our IR spectrometer, the calculations predict a weakly out-of-plane bending mode, which is IR-active at $181 \mathrm{~cm}^{-1}$ and is in very good agreement with the HREEL peak. As expected, this IRactive mode is not observed in fluorescence.

The next loss signal comes at about $259 \mathrm{~cm}^{-1}$. It appears broadened or split. Calculations predict an IR-active mode at $277 \mathrm{~cm}^{-1}$, but also, a totally symmetric (Raman- and fluorescence-active) mode at $246 \mathrm{~cm}^{-1}$. This Raman-mode corresponds to in-plane stretching of the whole molecule ${ }^{27}$ along its long axis (as shown in Figure 1d). We could not test whether the calculated IR-active mode appears in the IR spectrum, as our FTIR spectrograph cuts off at $300 \mathrm{~cm}^{-1}$. Therefore, the loss peak at $259 \mathrm{~cm}^{-1}$ could arise from either of these modes, or from both.

The next component is a weak and broad (width of $80 \mathrm{~cm}^{-1}$ ) loss peak at $547 \mathrm{~cm}^{-1}$. The corresponding region in the IR absorption spectrum shows multiple peaks at 492, 536, and 560 $\mathrm{cm}^{-1}$. The fluorescence spectrum also shows modes in that region at 496,542 , and $588 \mathrm{~cm}^{-1}$. Because of the lower spectral resolution in HREELS $\left( \pm 2.5 \mathrm{meV}= \pm 20 \mathrm{~cm}^{-1}\right)$ compared to that on IR absorption spectroscopy $\left( \pm 0.6 \mathrm{meV}= \pm 5 \mathrm{~cm}^{-1}\right)$, it is difficult to propose any clear assignment of this broad peak.

The most intense loss peak in the HREEL spectrum around $800 \mathrm{~cm}^{-1}$ is observed already after $2 \mathrm{~min}$ of exposure (Figure S2), at very low coverage. We can distinguish a weak shoulder at $690 \mathrm{~cm}^{-1}$, a more intense shoulder at $760 \mathrm{~cm}^{-1}$, and the most intense component of all our spectra at $815 \mathrm{~cm}^{-1}$. The IR spectrum (Figure 1b) shows three strong modes at 696, 754, and $808 \mathrm{~cm}^{-1}$. The calculated IR spectrum (Figure 1c) presents three modes around $800 \mathrm{~cm}^{-1}$, two of them correspond to outof-plane modes with frequencies at 765 and $829 \mathrm{~cm}^{-1}$ and one at $801 \mathrm{~cm}^{-1}$ for an in-plane mode (modes symmetry are shown in Table S1). Figure 2 shows the atomic displacements for those three modes ordered by increasing frequency. The fluorescence spectrum shows only one weak peak at $786 \mathrm{~cm}^{-1}$, possibly a combination of the long- and short-axis deformation modes, corresponding to $544+243 \mathrm{~cm}^{-1}$. No other active modes have ever been reported in this region from single molecule fluorescence spectra of terrylene in solid matrices. $^{28-32}$

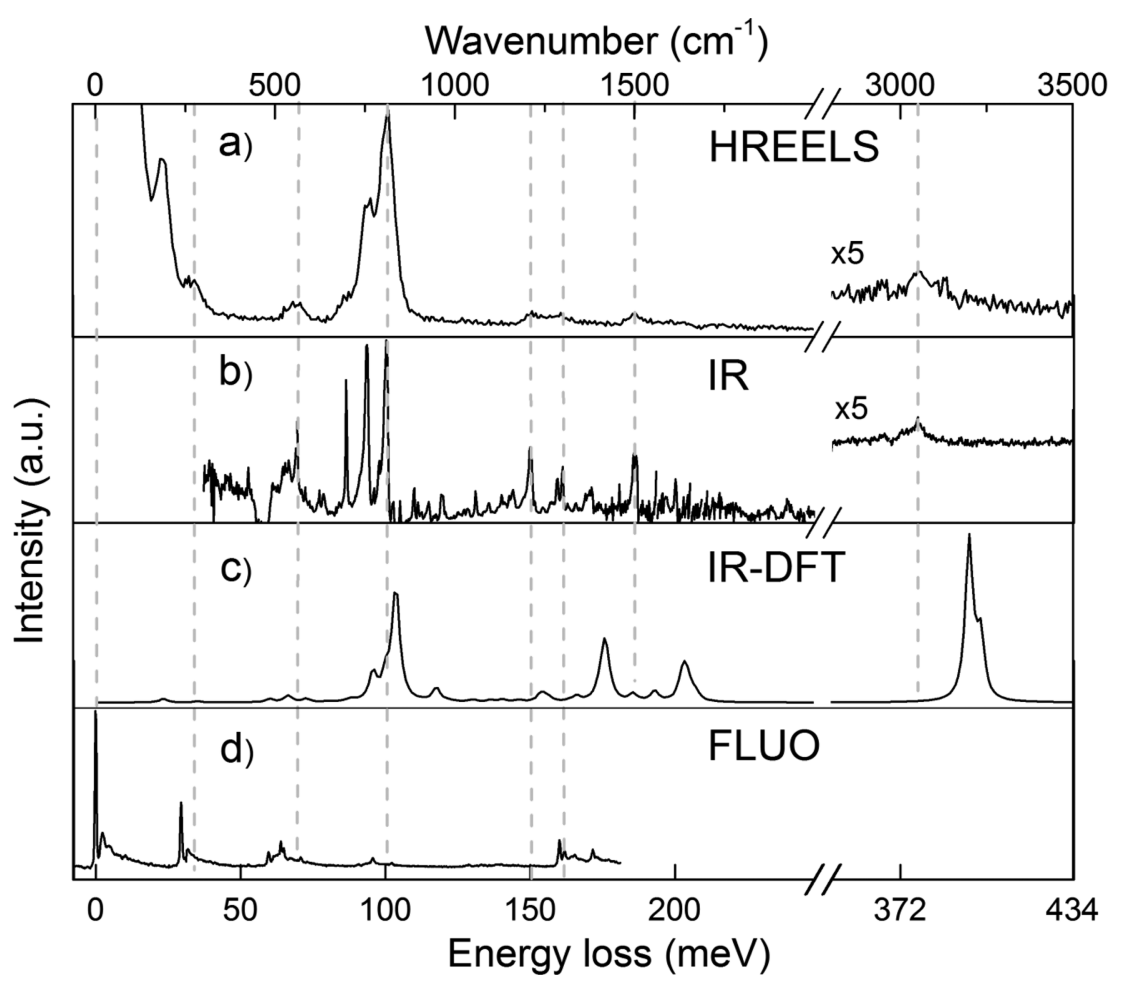

Figure 1. (a) Specular HREELS of a terrylene film (14 min) on $\mathrm{Au}(111)$ recorded with energy beam $E_{0}=5.0 \mathrm{eV}$ with $5 \mathrm{meV}$ resolution and $30 \mathrm{~min}$ acquisition time. (b) FTIR of terrylene in a $\mathrm{KBr}$ pellet. (c) Calculated IR spectrum considering a fwhm $=25 \mathrm{~cm}^{-1}$. (d) Fluorescence spectrum from one single terrylene molecule at $1.5 \mathrm{~K}$ (the zero position corresponds to the zero-phonon line (ZPL) at $577.13 \mathrm{~nm}$ or $17327 \mathrm{~cm}^{-1}$ ). The dotted lines are guides to follow the peak positions found in each spectrum. 

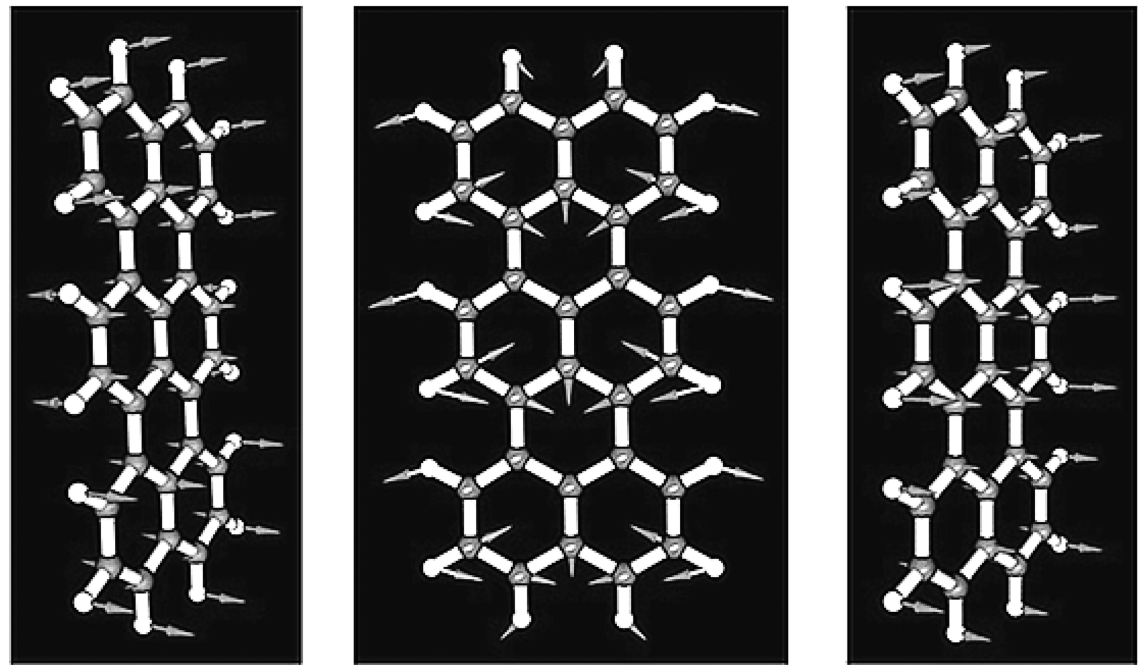

Figure 2. Atomic displacements calculated for molecular vibrations of terrylene at 765,801 , and $829 \mathrm{~cm}^{-1}$, from left to right. Those modes might be the ones responsible for the strongest loss peak in EELS experiments. Atomic displacements were obtained based on the DFT B3LYP/6-31G(d,p) method.

Table 1. Frequencies (in $\mathrm{cm}^{-1}$ ) of the Main Vibrational Modes of Terrylene Found in HREELS on the Au(111) Surface, Measured on Terrylene in a KBr pellet by FTIR, Calculated IR and Fluorescence (or Raman), and in a Single-Molecule Fluorescence Spectrum ${ }^{23}$ (SMS), Respectively ${ }^{a}$

\begin{tabular}{|c|c|c|c|c|c|}
\hline mode assignment & HREELS $\left(\mathrm{cm}^{-1}\right)$ & $\operatorname{IR}\left(\mathrm{cm}^{-1}\right)$ & IR-DFT $\left(\mathrm{cm}^{-1}\right)$ & Fluo-DFT $\left(\mathrm{cm}^{-1}\right)$ & $\operatorname{SMS}\left(\mathrm{cm}^{-1}\right)$ \\
\hline out-of-plane bending & 185 & - & 181 & no active modes & not present \\
\hline long-axis stretching & 259 & - & 277 & 246 & 249 \\
\hline \multirow[t]{3}{*}{ short-axis stretching } & 547 & 492 & 478 & 447 & 496 \\
\hline & & 536 & 528 & 544 & 522 \\
\hline & & 560 & 579 & 590 & 588 \\
\hline \multirow[t]{3}{*}{ out-of-plane $\mathrm{C}-\mathrm{H}$ wagging } & 690 & 696 & 765 & no active modes & very weak $544+246$ \\
\hline & 760 & 754 & 801 & & \\
\hline & 815 & 808 & 829 & & \\
\hline \multirow[t]{2}{*}{ in-plane $\mathrm{C}-\mathrm{H}$ bending } & 1200 & 1208 & 1232 & no active modes & not detected \\
\hline & 1280 & 1298 & 1256 & & \\
\hline \multirow[t]{6}{*}{ aromatic $\mathrm{C}=\mathrm{C}$ stretching } & & & & 1307 & 1272 \\
\hline & & & & 1343 & 1287 \\
\hline & & & & 1391 & 1313 \\
\hline & & & 1402 & 1403 & 1357 \\
\hline & 1495 & 1496 & 1491 & & \\
\hline & & 1612 & 1631 & & \\
\hline $\mathrm{C}-\mathrm{H}$ stretching & 3054 & 3065 & 3200 & not reported & - \\
\hline
\end{tabular}

${ }^{a_{T}}$ The experimental errors in the determination of the frequencies are $\pm 10 \mathrm{~cm}^{-1}$ in HREELS, $\pm 5 \mathrm{~cm}^{-1}$ in FTIR, $\pm 3 \mathrm{~cm}^{-1}$ in fluorescence. The complete set of molecular vibrations for terrylene in the gas phase, found with the DFT B3LYP/6-31G(d,p) method, are shown in Table S1. Dotted lines indicate an experimental limitation of the equipment.

The next HREELS signals at 1200 and $1280 \mathrm{~cm}^{-1}$ are weak but appear consistently even for very thin films. The corresponding FTIR spectrum presents maxima at 1208 and $1298 \mathrm{~cm}^{-1}$. According to calculations these modes correspond to $\mathrm{C}-\mathrm{H}$ in-plane wagging. Because the displacements are parallel to the metallic surface, their intensity should be strongly reduced by the electrostatic image effect (Chart 1 , right side). In fluorescence, another four in-plane modes appear at higher energies 1272, 1287, 1313, and $1357 \mathrm{~cm}^{-1}$, which are presumably visible in Raman spectra too.

The next energy interval around $1500 \mathrm{~cm}^{-1}$ corresponds to the stretching of the carbon-carbon aromatic bonds. Only one band appears at $1495 \mathrm{~cm}^{-1}$ in the HREEL spectrum (Figure 1a). The calculations predict several modes (the complete series, with symmetries and energies can be found in Table S1 of the Supporting Information) within $1300-1600 \mathrm{~cm}^{-1}$. Two of them with a significant IR activity are predicted, at 1402 and $1631 \mathrm{~cm}^{-1}$; however a weak one at $1491 \mathrm{~cm}^{-1}$ matches the HREEL signal. The measured IR spectrum shows an intense mode at $1496 \mathrm{~cm}^{-1}$ in agreement with HREELS and calculations. The fluorescence spectrum does not extend to this frequency area because of the upper limit of our spectrometer.

Finally, the HREELS shows a weak vibration at $3054 \mathrm{~cm}^{-1}$, which can be attributed to a $\mathrm{C}-\mathrm{H}$ stretching $\nu(\mathrm{C}-\mathrm{H})$. This is in good agreement with the FTIR spectrum where this mode appears at $3065 \mathrm{~cm}^{-1}$. However, the DFT calculation finds this mode at $3200 \mathrm{~cm}^{-1}$. For comparison with experiments we used different scaling factors to improve the correspondence. For B3LYP/6-31G $(d, p)$ basis the scaling factor varies from 0.97 to 
1.0. As a result, for high-frequency vibrations such as $\mathrm{C}-\mathrm{H}$ stretching, this factor plays a bigger role than in the case of lower-energy modes.

Those modes that were found to correspond with experimental observations are shown in Table 1 (the complete set of molecular vibrations can be found in the Table S1, in the Supporting Information). On the whole, we notice a fair agreement between the HREEL spectrum and the measured FTIR spectrum, both in position and relative intensity of the bands. It is likely that the HREELS peaks with the highest intensities correspond to out-of-plane modes enhanced by the electrostatic image effect discussed in Chart 1. Nonetheless, some in-plane modes according to our assignment are visible in the HREEL spectrum and therefore are not completely suppressed by the electrostatic image effect. This may arise from a different excitation mechanism or from molecules that are not lying completely flat on the surface. Some of the differences in frequencies can arise from the different environments of the molecules, the gold surface in HREELS and the microcrystal environment in the IR $\mathrm{KBr}$ pellet measurement.

We now give a brief discussion of the vibrational modes of terrylene. An isolated terrylene molecule belongs to the symmetry group $D_{2 h}$. This group has only one-dimensional representations. Accordingly, all vibrational modes of the molecule are nondegenerate and can be found in Table S1 in the Supporting Information. The molecule presents 132 vibrational modes from which 56 are infrared-active (representations $\left.B_{1 \mathrm{w}}, B_{2 \mathrm{w}}, B_{3 \mathrm{u}}\right), 66$ are Raman-active $\left(A_{\mathrm{g}}, B_{1 \mathrm{~g}}, B_{2 \mathrm{~g}}, B_{3 \mathrm{~g}}\right)$, and the other 10 modes are silent (representation $A_{u}$ ).

3.2. HREEL Spectrum at Increasing Exposition Times. Figure 3 shows HREELS spectra for increasing exposure times

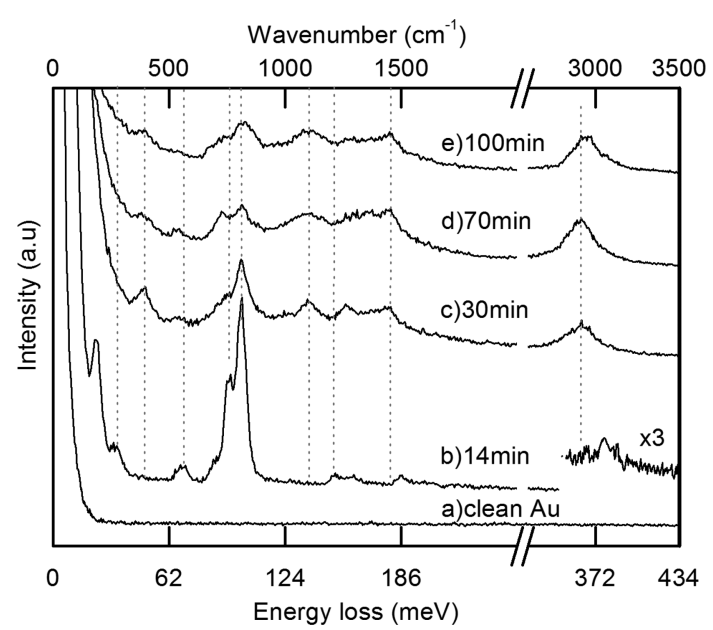

Figure 3. Specular HREEL spectra for increasing terrylene film thickness on top of $\mathrm{Au}(111)$. The lowest spectrum is the baseline obtained from a clean $\mathrm{Au}(111)$ surface before deposition. The dotted lines are guides to help to follow the peak evolution. Each spectra was recorded with energy beam $E_{0}=5.0 \mathrm{eV}$ with $5 \mathrm{meV}$ resolution and 30 min acquisition time.

14, 30, 70, and $100 \mathrm{~min}$. These spectra display clear changes. The most striking feature in these spectra is the relative intensity decrease of the modes around $800 \mathrm{~cm}^{-1}$, which we assigned to out-of-plane $\mathrm{C}-\mathrm{H}$ wagging. The decrease in intensity could arise from a different structure of the molecules at the surface or from a change in interaction with the surface.
A second feature is the appearance of two new modes for larger thicknesses, at $400 \mathrm{~cm}^{-1}$ and around $1090 \mathrm{~cm}^{-1}$. The first one becomes visible after $30 \mathrm{~min}$ and stays unchanged at longer exposure times. The second one is in the aromatic stretching area, and it could appear because of a change in orientation of the terrylene molecules when the film becomes thicker.

Finally, we observe a shift of the $\mathrm{C}-\mathrm{H}$ stretch from 3054 to $2920 \mathrm{~cm}^{-1}$. It is not possible to ensure that these peaks correspond to the same molecular vibration because of the big frequency mismatch. But in case they are the same, the direct change of the electrostatic interaction with the gold surface as the film becomes thicker could explain the frequency shift. Alternatively, a different $\mathrm{C}-\mathrm{H}$ stretch mode could be excited at high thickness because of a different molecular orientation.

The HREEL spectra at early deposition times with submonolayer coverage (shown in the Supporting Information, Figure S2) very probably correspond to molecules lying almost flat on the surface. Our assignment of the strong modes at low coverage is therefore rather safe. At higher coverage, however, we believe that a Stranski-Krastanov type of growth takes over and that three-dimensional aggregates, with non-flat molecular orientations, appear. A better understanding of the weak modes in the HREEL spectra will thus require knowledge of the structure of the deposited films as a function of thickness, and in particular determination of the orientation of the molecules.

3.3. EELS Probing of Electronic Excitations of Terrylene. Figure 4 shows the HREEL spectrum of terrylene

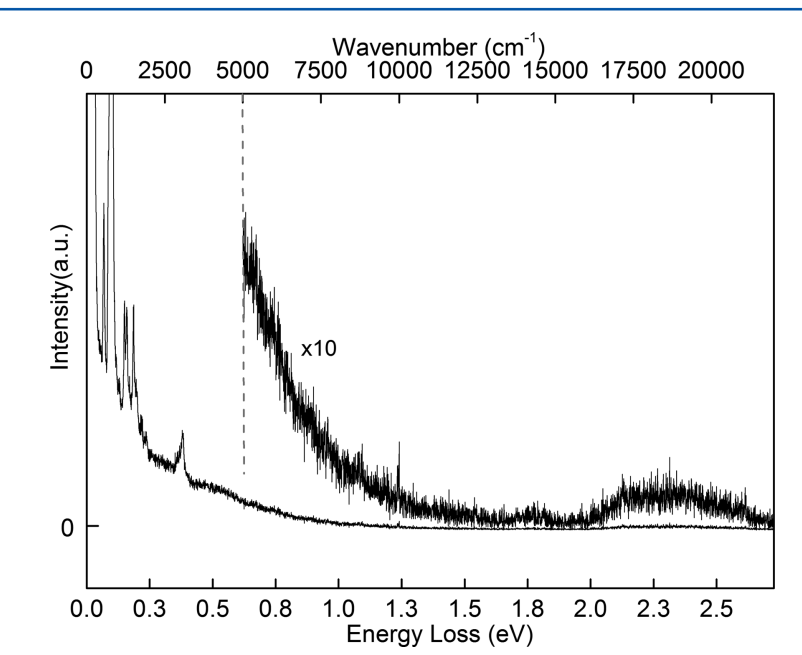

Figure 4. EELS probing of electronic excitations of terrylene. The spectrum was recorded with energy beam $E_{0}=12 \mathrm{eV}$, with $5 \mathrm{meV}$ resolution and $30 \mathrm{~min}$ acquisition time after $2 \mathrm{~h}$ of exposure on the Au-surface to the terrylene (Knudson cell).

thick films (more than 20 monolayers) for the complete spectral range of losses between 0 and $22000 \mathrm{~cm}^{-1}$. Intense peaks are clear in the vibrational region between $0-4000 \mathrm{~cm}^{-1}$ (as discussed in previous sections). Then, it is also possible to distinguish a clear and broad signal in the high-energy loss region between 16000 and $20000 \mathrm{~cm}^{-1}(2.0$ and $2.5 \mathrm{eV})$, which is centered at $18147 \mathrm{~cm}^{-1}(2.25 \mathrm{eV})$. Looking in more detail into this broad feature it is possible to see a maximum loss signal around $2.1 \mathrm{eV}$. This very broad feature must be composed of several peaks, but at least it is possible to distinguish a main loss signal around $2.1 \mathrm{eV}$. This is confirmed by optical spectroscopy as shown in the Supporting Information (Figure S3) and therefore can be assigned to the 
singlet-to-singlet electronic transition. Even further, the other component at $18550 \mathrm{~cm}^{-1}(2.3 \mathrm{eV})$ agrees with the expected vibrational-electronic transition found in the optical spectrum (Figure S3). Next, we look for the spin-forbidden singlet-totriplet electronic transition of terrylene. This transition has been predicted by quantum chemical calculations to lie at about $8000 \pm 800 \mathrm{~cm}^{-1}(1 \pm 0.1 \mathrm{eV})$. However, as can be seen in Figure 4, no clear signal appears around this interval in the energy loss spectra. It is possible to see a weak feature at $1.8 \mathrm{eV}$. However, the energy mismatch between prediction and observation does not allow us yet to do the assignment.

\section{CONCLUSIONS}

HREELS is a surface-sensitive technique that provides information about modes inaccessible by optical means. The selection rules and excitation mechanisms of HREELS are thus complementary to optical spectroscopy. We have succeeded in electronically exciting terrylene to the first excited-singlet state using the HREELS electron beam, but the spin-forbidden transition toward the lowest triplet state remains unknown. The strongest terrylene vibration modes in HREELS appearing around $800 \mathrm{~cm}^{-1}$ have never been reported before from singlemolecule fluorescence spectroscopy. The HREEL spectrum agrees fairly well with the IR absorption. With support from calculations, we assign the two strongest modes to $\mathrm{C}-\mathrm{H}$ out-ofplane wagging (Figure 2). To progress in the assignment of the other HREELS modes, delicate structural studies in the submonolayer regime are the natural next steps of this work.

More broadly, we have shown that terrylene is a suitable molecule for controlled deposition in an UHV system. We were able to produce organic films on $\mathrm{Au}(111)$ which were stable for many hours of exposure to the electron beam and to X-rays. Terrylene shows a good compatibility and weak interactions with gold, which may prove interesting for future organic electronics devices.

\section{ASSOCIATED CONTENT}

\section{S Supporting Information}

We show the decreasing X-ray photoelectron (XPS) signal from gold- $4 \mathrm{f}_{7 / 2}$ electrons and the increasing signal of carbon- $1 \mathrm{~s}$ electrons. The XPS data helped to optimize the deposition conditions and to qualitatively determine the amount of terrylene deposited in the Au-surface. In Figure S1c, both intensities were normalized to the clean $\mathrm{Au}-4 \mathrm{f}_{7 / 2}$ signal before deposition. The HR-EELS spectra in Figure 3 and in S2, where taken always after the XP spectra. The optical absorption of a terrylene solution is compared in S3 to the loss signal found in Figure 4. The vibrational energies and the symmetries of vibrational modes calculated by DFT are given in Table S1. This material is available free of charge via the Internet at http://pubs.acs.org.

\section{AUTHOR INFORMATION}

\section{Corresponding Author}

*Tel.: (+31) (0)71 527 1720/5910. Fax: (+31) (0)71 527 5819. E-mail: orrit@physics.leidenuniv.nl.

\section{Notes}

The authors declare no competing financial interest.

\section{ACKNOWLEDGMENTS}

We would like to thank Prof. Boleslaw Kozankiewicz, from the Institute of Physics, Polish Academy of Science, Warsaw,
Poland, for the intense discussions. We also thank Dr. Andreas Köhn, from JGU, Theoretical Chemistry Department on Mainz, who predicted the triplet-state energy of terrylene by also using DFT calculations. We thank Dr. Carlos Sabatér for the visualization of terrylene on the gold surface, atomic and molecular conductors group in Leiden University. The DFT calculations in this work were performed at the Interdisciplinary Center of Mathematical and Computer Modeling (ICM) of Warsaw University under Computational Grant G-32-10. Special thanks are due to Anne-Marie Franken in Jülich for help with the HREELS experiments. This work was supported by the Stichting Fundamenteel Onderzoek der Materie (FOM) Project No. L2105, which is part of the Nederlandse Organisatie voor Wetenschappelijk Onderzoek (NWO).

\section{REFERENCES}

(1) Ibach, H.; Balden, M.; Bruchmann, D.; Lehwald, S. Electronenergy loss spectroscopy - recent advances in technology and application. Surf. Sci. 1992, 269, 94-102.

(2) Tautz, F. S. Structure and bonding of large aromatic molecules on noble metal surfaces: the example of PTCDA. Prog. Surf. Sci. 2007, 82, 479-520.

(3) Nakamura, T.; Iwasawa, K.; Kera, S.; Azuma, Y.; Okudaira, K. K.; Ueno, N. Low-energy molecular exciton in indium/perylene-3,4,9, 10tetracarboxylic dianhydride system observed by electronic energy loss spectroscopy. Appl. Surf. Sci. 2003, 212, 515-519.

(4) Shklover, V.; Tautz, F. S.; Scholz, R.; Sloboshanin, S.; Sokolowski, M.; Schaefer, J. A.; Umbach, E. Differences in vibronic and electronic excitations of PTCDA on $\operatorname{Ag}(111)$ and $\operatorname{Ag}(110)$. Surf. Sci. 2000, 454, $60-66$.

(5) Bulovic, V.; Burrows, P. E.; Forrest, S. R.; Cronin, J. A.; Thompson, M. E. Study of localized and extended excitons in 3,4,9,10perylenetetracarboxylic dianhydride (PTCDA): Spectroscopic properties of thin films and solutions. Chem. Phys. 1996, 210, 1-12.

(6) Swiderek, P.; Michaud, M.; Hohlneicher, G.; Sanche, L. Electronenergy loss spectroscopy of solid naphthalene and acenaphthene search for the low-lying triplet-states. Chem. Phys. Lett. 1990, 175, 667-673.

(7) Haas, U.; Thalacker, C.; Adams, J.; Fuhrmann, J.; Riethmuller, S.; Beginn, U.; Ziener, U.; M?ller, M.; Dobrawa, R.; Wurthner, F. Fabrication and fluorescence properties of perylene bisimide dye aggregates bound to gold surfaces and nano patterns. J. Mater. Chem. 2003, 13, 767-772.

(8) Winder, C.; Matt, G.; Hummelen, J. C.; Janssen, R. A. J.; Sariciftci, N. S.; Brabec, C. J. Sensitization of low bandgap polymer bulk heterojunction solar cells. Thin Solid Films 2002, 403, 373-379.

(9) Kudo, K.; Iizuka, M.; Kuniyoshi, S.; Tanaka, K. Device characteristics of lateral and vertical type organic field effect transistors. Thin Solid Films 2001, 393, 362-367.

(10) Wood, S.; Kim, J. S.; James, D. T.; Tsoi, W. C.; Murphy, C. E.; Kim, J. S. Understanding the relationship between molecular order and charge transport properties in conjugated polymer based organic blend photovoltaic devices. J. Chem. Phys. 2013, 139, 7-19.

(11) Karl, N. Charge carrier transport in organic semiconductors. Synth. Met. 2003, 133, 649-657.

(12) Toda, Y.; Yanagi, H. Electroluminescence of epitaxial perylene films. Appl. Phys. Lett. 1996, 69, 2315-2317.

(13) Kazmaier, P. M.; Hoffmann, R. A Theoretical-study of crystallochromy - quantum interference effects in the spectra of perylene pigments. J. Am. Chem. Soc. 1994, 116, 9684-9691.

(14) Komolov, S.; Aliaev, Y. Influence of the substrate properties on the electronic structure of organic film-inorganic substrate interfaces. Technol. Phys. 2007, 52, 1163-1168.

(15) Barlow, S. M.; Raval, R. Complex organic molecules at metal surfaces: bonding, organisation and chirality. Surf. Sci. Rep. 2003, 50, 201-341. 
(16) Kosciesza, R.; Luzina, E.; Wiacek, D.; Dresner, J.; Kozankiewicz, B. Photo-stability of single terrylene molecules in 2,3-dimethylnaphthalene crystals. Mol. Phys. 2009, 107, 1889-1895.

(17) Wirtz, A. C.; Dokter, M.; Hofmann, C.; Groenen, E. J. J. Spincoated polyethylene films for single-molecule optics. Chem. Phys. Lett. 2006, 417, 383-388.

(18) Unwin, P. J.; Jones, T. S. Vibrational properties of ordered perylene thin films on $\operatorname{GaAs}(100)$ and $\operatorname{InAs}(111) A$. Surf. Sci. 2003, 532, 1011-1016.

(19) Gao, L.; Sun, J.; Cheng, Z.; Deng, Z.; Lin, X.; Du, S.; Gao, X. H. Structural evolution at the initial growth stage of perylene on $\mathrm{Au}(111)$. Surf. Sci. 2007, 601, 3179-3185.

(20) Lee, S. K.; Zu, Y. B.; Herrmann, A.; Geerts, Y.; Mullen, K.; Bard, A. J. Electrochemistry, spectroscopy and electrogenerated chemiluminescence of perylene, terrylene, and quaterrylene diimides in aprotic solution. J. Am. Chem. Soc. 1999, 121, 3513-3520.

(21) Orrit, M. Nano-optics - quantum light switch. Nat. Phys. 2007, $3,755-756$.

(22) Orrit, M.; Bernard, J.; Zumbusch, A.; Personov, R. I. Stark-effect on single molecules in a polymer matrix. Chem. Phys. Lett. 1992, 196, 595-600.

(23) Navarro, P.; Tian, Y.; van Stee, M.; Orrit, M. Stable singlemolecule lines of terrylene in polycrystalline para-dichlorobenzene at 1.5 K. ChemPhysChem 2014, 15, 3032-3039.

(24) Barth, J. V.; Brune, H.; Ertl, G.; Behm, R. J. Scanning tunneling microscopy observations on the reconstructed $\mathrm{Au}(111)$ surface atomic-structure, long-range superstructure, rotational domains, and surface-defects. Phys. Rev. B 1990, 42, 9307-9318.

(25) Frisch, M. J.; Trucks, G. W.; Schlegel, H. B.; Scuseria, G. E.; Robb, M. A.; Cheeseman, J. R.; Scalmani, G.; Barone, V.; Mennucci, B.; Petersson, G. A.; et al. Gaussian 09, Revision B.01; Gaussian Inc.: Wallingford, 2010.

(26) Barone, V.; Bloino, J.; Biczysko, M.; Santoro, F. Fully integrated approach to compute vibrationally resolved optical spectra: from small molecules to macrosystems. J. Chem. Theory Comput. 2009, 5, 540554.

(27) Deperasinska, I.; Zehnacker, A.; Lahmani, F.; Borowicz, P.; Sepiol, J. Fluorescence studies of terrylene in a supersonic jet: indication of a dark electronic state below the allowed transition. $J$. Phys. Chem. A 2000, 111, 4252-4258.

(28) Tchenio, P.; Myers, A. B.; Moerner, W. E. Vibrational analysis of the dispersed fluorescence from single molecules of terrylene in polyethylene. Chem. Phys. Lett. 1993, 213, 325-332.

(29) Kummer, S.; Basche, T.; Brauchle, C. Terrylene in paraterphenyl - a novel single-crystalline system for single-molecule spectroscopy at low-temperatures. Chem. Phys. Lett. 1994, 229, 309316.

(30) Kozankiewicz, B.; Bernard, J.; Orrit, M. Single-molecule lines and spectral hole-burning of terrylene in different matrices. J. Chem. Phys. 1994, 101, 9377-9383.

(31) Palewska, K.; Lipinski, J.; Sworakowski, J.; Sepiol, J.; Gygax, H.; Meister, E. C.; Wild, U. P. Total luminescence spectroscopy of terrylene in low-temperature Shpolskii matrices. J. Phys. Chem. 1995, 99, 16835-16841.

(32) Deperasinska, I.; Kozankiewicz, B.; Biktchantaev, I.; Sepiol, J. Anomalous fluorescence of terrylene in neon matrix. J. Phys. Chem. A 2001, 105, 810-814. 